\title{
Minimal flavour violation and SU(5)-unification
}

\author{
Riccardo Barbieri ${ }^{\mathrm{a}}$, Fabrizio Senia ${ }^{\mathrm{b}}$ \\ Scuola Normale Superiore and INFN, Piazza dei Cavalieri 7, 56126 Pisa, Italy
}

Received: 8 October 2015 / Accepted: 26 November 2015 / Published online: 17 December 2015

(C) The Author(s) 2015. This article is published with open access at Springerlink.com

\begin{abstract}
Minimal flavour violation in its strong or weak versions, based on $U(3)^{3}$ and $U(2)^{3}$, respectively, allows suitable extensions of the standard model at the TeV scale to comply with current flavour constraints in the quark sector. Here we discuss considerations analogous to minimal flavour violation (MFV) in the context of $S U(5)$-unification, showing the new effects/constraints that arise both in the quark and in the lepton sector, where quantitative statements can be made controlled by the CKM matrix elements. The case of supersymmetry is examined in detail as a particularly motivated example. Third generation sleptons and neutralinos in the few hundred $\mathrm{GeV}$ range are shown to be compatible with current constraints.
\end{abstract}

\section{Introduction}

The discovery of the Higgs boson and the measurement of some of its couplings, together with the number of different flavour measurements performed in the last 15 years or so, have raised the tests of the standard model (SM) to a qualitatively higher level. On one side there is the reported evidence for a linear relation, $m_{i}=\lambda_{i} v$, between the masses and the couplings to the Higgs boson of the $\mathrm{SM}$ particles (for the moment the heavier ones). On the other side several of the flavour-changing SM loops have been experimentally confirmed with strengths as expected in the Cabibbo-Kobayashi-Maskawa (CKM) picture of flavour physics. Altogether it is appropriate to say that the ensemble of these tests have turned the Yukawa couplings of the Higgs boson in the SM into an element of physical reality. At the same time this strikingly underlines what is perhaps the major weakness of the SM itself: its inability to predict any of these couplings. This is the current status of the flavour problem in the SM, which strongly motivates the efforts to

\footnotetext{
a e-mail: barbieri@sns.it

b e-mail: fabrizio.senia@sns.it
}

increase the precision of the mentioned tests, now typically at the $10 \div 30 \%$ level.

When trying to go beyond the SM, the description of flavour faces a further problem of different nature. If new particles are expected at the $\mathrm{TeV}$ scale, the compliance with the flavour tests is highly non trivial. Attempts to achieve it rest on dynamical assumptions, on flavour symmetries or on a combination thereof. Examples of the first kind are gauge or anomaly mediation of supersymmetry breaking, whereas a combinations of symmetries (typically $U(1)$ factors) and dynamics is invoked in models of alignment. Based on symmetries alone, minimal flavour violation (MFV) is the way to make new physics at the TeV scale compatible with flavour tests. By MFV phenomenologically defined we mean here that in an Effective Field Theory (EFT) approach the only relevant operators are the ones that correspond to the Flavour Changing Neutral Current effects occurring in the SM, weighted by a common scale and by the standard CKM factors up to (possibly flavour dependent) coefficients of order unity. As briefly recalled in the next section there exists a strong version of MFV [2-4] based on the $U(3)^{3}$ flavour group (or equivalent) and a weak version, based on the $U(2)^{3}$ flavour group [5,6] (or equivalent).

$\mathrm{MFV}$, as recalled above, refers to the quark sector. Is there something analogous that can be said on the lepton sector, always having in mind new physics not far from the TeV scale? When asking such question, what comes immediately to mind is the issue of neutrino masses, whose nature (Dirac or Majorana) and origin (at low or high energy among other issues) are unknown. ${ }^{1}$ This is a difficulty. Perhaps the very small neutrino masses do not influence at all the flavour structure of the charged lepton sector. If so, however, what is left that can possibly constrain it? In the SM, without neutrino masses, one remains with individual lepton number conservation. With extra particles at the TeV scale individual lepton number conservation is unlikely, but, leaving out neutrino

\footnotetext{
${ }^{1}$ For some early works extending MFV to the lepton sector see [7-10].
} 
masses, one seems to lose any way to argue further in a truly quantitative way.

In this paper we discuss to what extent $S U(5)$ unification can avoid this impasse. By $S U(5)$ unification we simply mean that there exist definite $S U(5)$-invariant Yukawa couplings that give rise, after symmetry breaking, to realistic quark masses and mixings as well as to the observed charged lepton masses. In the low energy theory this leads both to deviations from MFV in the quark sector and to a definite pattern of flavour violation in the charged lepton sector, always controlled by the CKM matrix elements. The compatibility of such patterns with current bounds will be discussed in general as well as, in particular, considering the possible existence of supersymmetric particles at the $\mathrm{TeV}$ scale.

The relation between strong MFV and $S U(5)$-unification has been first discussed in [1]. In this paper we analyse the relation of $S U(5)$-unification with both versions of $\mathrm{MFV}$, strong or weak, as defined above, pointing out specific differences between the two cases.

\section{Strong and weak minimal flavour violation}

Strong MFV [2-4] is obtained by assuming the quark flavour symmetry in the gauge sector of the SM

$$
U(3)^{3} \equiv U(3)_{Q} \times U(3)_{u} \times U(3)_{d}
$$

to be as well a symmetry of whatever beyond the standard model (BSM) theory under examination, only broken by the standard Yukawa coupling matrices $Y_{u}$ and $Y_{d}$ in the directions

$Y_{u}=(3, \overline{3}, 1) \quad Y_{d}=(3,1, \overline{3})$

Although this gives up any attempt to understand the pattern of quark masses and mixings, it nevertheless leads to MFV since, by symmetry transformations, $Y_{u}$ and $Y_{d}$ can be reduced as in the SM to the form

$Y_{u}=Y_{u}^{D}, \quad Y_{d}=V Y_{d}^{D}$

where $Y_{u, d}^{D}$ are diagonal and $V$ is the CKM matrix. Here with $Y_{u}$ and $Y_{d}$ we denote the low energy Yukawa couplings with canonically normalised quark fields, which in general differ from the original symmetry-breaking parameters but have necessarily the same transformation properties under $U(3)^{3}$ and can equally well be used to characterise the symmetry breaking in the EFT.

Weak MFV $[5,6]$ is based on the observation that

$U(2)_{Q} \times U(2)_{u} \times U(2)_{d} \times U(1)_{d 3}$, briefly called $U(2)^{3}$, is an approximate symmetry of the quark spectrum and mixings. ${ }^{2}$ This suggests to consider $U(2)^{3}$ rather than $U(3)^{3}$ as the relevant symmetry with $U(2)^{3}$ breaking described by small parameters. The only minimal set of spurions that can do this is

$$
\begin{aligned}
y_{d 3} & =(1,1,1)_{-1} \quad \Delta_{u}=(2, \overline{2}, 1)_{0} \quad \Delta_{d}=(2,1, \overline{2})_{0} \\
\mathbf{V} & =(2,1,1)_{0}
\end{aligned}
$$

The smallness of these parameters relates to the smallness of all quark Yukawa couplings except the top one and to the smallness of the elements $V_{t d}, V_{t s}, V_{u b}, V_{c b}$ of the CKM matrix. The presence of a doublet, possibly different from $\mathbf{V}$, is necessary to allow the communication between the third and the first two generations. It is simple to convince oneself that any other single doublet transforming differently from $\mathbf{V}$ would have to be of order unity, thus strongly breaking $U(2)^{3}$, and would not lead to MFV.

The low energy Yukawa couplings acquire the form

$Y_{u}=\left(\begin{array}{c:c:c}\lambda_{u} & y_{t} x_{t} \mathbf{V} \\ \hdashline 0 & y_{t} .\end{array}\right) \quad Y_{d}=\left(\begin{array}{c:c}\lambda_{d} & y_{b} x_{b} \mathbf{V} \\ \hdashline 0 & y_{b}\end{array}\right)$

Here $x_{t, b}$ are order one coefficients and, by symmetry transformations, one can set

$\mathbf{V}=\left(\begin{array}{l}0 \\ \epsilon\end{array}\right), \quad \lambda_{u}=L_{12}^{u} \lambda_{u}^{D}, \quad \lambda_{d}=\Phi_{L} L_{12}^{d} \lambda_{d}^{D}$,

where $\epsilon$ is a real parameter, $L_{12}^{u, d}$ are rotation matrices in the space of the first two generations with angles $\theta_{L}^{u, d}$ and $\Phi_{L}=$ $\operatorname{diag}\left(e^{-i \phi}, 1\right)$, i.e. four parameters in total. Due to the smallness of $\epsilon, \lambda_{u}$ and $\lambda_{d}$, both $Y_{u}$ and $Y_{d}$ are perturbatively diagonalised by pure left transformations, $Y_{u}=U_{u} Y_{u}^{D}, Y_{d}=$ $U_{d} Y_{d}^{D}$ with $U_{u, d}$ both of the form $U=U_{23} U_{12}$, i.e. the products of two successive unitary transformations in the 12 and 2-3 sectors. In turn this gives for the standard CKM matrix

$V_{C K M}=U_{u}^{+} U_{d}=\left(\begin{array}{ccc}c_{L}^{u} c_{L}^{d} & \lambda & s_{L}^{u} s e^{-i \delta} \\ -\lambda & c_{L}^{u} c_{L}^{d} & c_{L}^{u} s \\ -s_{L}^{d} s e^{i(\delta+\phi)} & -c_{L}^{d} s & 1\end{array}\right)$,

where $s \sim O(\epsilon), s_{L}^{u, d}=\sin \theta_{L}^{u, d}, c_{L}^{u, d}=\cos \theta_{L}^{u, d}$ and $s_{L}^{u} c_{L}^{d}-s_{L}^{d} c_{L}^{u} e^{i \phi}=\lambda e^{i \delta}$. Using this parametrisation of the CKM matrix, a direct fit of the tree-level flavour observables, presumably not influenced by new physics, results in

$$
\begin{aligned}
s_{L}^{u} & =0.096 \pm 0.008, & s_{L}^{d} & =-0.215 \pm 0.011, \\
s & =0.0411 \pm 0.0010, & \phi & =(95 \pm 7)^{\circ} .
\end{aligned}
$$

\footnotetext{
${ }^{2}$ In fact a larger approximate symmetry of the SM is $U(2)_{Q} \times U(2)_{u} \times$ $U(3)_{d}[11]$. However, implementing MFV with this symmetry leads exactly to the same patterns as $U(3)^{3}[12]$.
} 
This determines the left transformations that diagonalise $Y_{u}$ and $Y_{d}$, up to the order one coefficients $x_{t}, x_{b}$, and leads to phenomenological MFV. As in the $U(3)^{3}$ case, the only relevant flavour-changing operators are the same as in the SM with coefficients controlled by the CKM factors $\xi_{i j}=V_{t i} V_{t j}^{*}$. Unlike the $U(3)^{3}$ case (strong MFV), however, between the $\Delta B=1,2$ and the $\Delta S=1,2$ transitions there are relative $\mathscr{O}(1)$ coefficients, generally complex (weak MFV).

\section{$3 U(3)^{2}$ and $S U(5)$-unification}

In terms of the usual trinification of $10(T) \oplus \overline{5}(\bar{F})$ representations of $S U(5)$, the minimal set of Yukawa couplings for realistic charged fermion masses is

$\mathscr{L}_{Y}^{U(3)}=T Y_{u} T H_{5}+T Y_{1} \bar{F} H_{\overline{5}}+T Y_{2} \bar{F} H_{45}$

where $H_{5}, H_{5}, H_{45}$ are Higgs fields transforming under $S U(5)$ as indicated, each with an $S U(2) \times U(1)$ breaking component of similar size, and flavour indices are everywhere left understood. The inclusion of a coupling to $H_{45}$ is necessary to account for the different $\mu-s$ and $e-d$ masses. A possibility to describe neutrino masses is to introduce a triplet of fermions, $N$, not transforming under $S U(5)$ and include in $\mathscr{L}_{Y}$ the further terms $N Y_{N} \bar{F} H_{5}+N M N$. We assume that the elements of $Y_{N}$ are small enough not to influence the considerations developed in the following. This is certainly consistent, e.g., if any of the elements of the matrix $M$ is less than $10^{11} \mathrm{GeV}$. As mentioned, we do this to limit the impact of our ignorance on the values of $Y_{N}$ and $M$ separately.

In analogy with strong MFV in the SM, the obvious way to go in the $S U(5)$ case is to consider the symmetry

$U(3)_{T} \times U(3)_{\bar{F}} \equiv U(3)^{2}$,

which acts on $T$ and $\bar{F}$ as $T=(3,1)$ and $\bar{F}=(1,3)$ [1]. Furthermore one assumes that $U(3)^{2}$ is only broken in the directions

$Y_{u}=(\overline{6}, 1), \quad Y_{1}=(\overline{3}, \overline{3}), \quad Y_{2}=(\overline{3}, \overline{3})$,

no matter which other operator is present at whatever scale.

At the TeV scale, after integrating out all heavy particles and including RGE running effects, the Yukawa Lagrangian, written in conventional notation, takes the form

$\mathscr{L}_{\text {low energy }}^{U(3)}=\bar{Q}_{L} \lambda_{u} u_{R} h+\bar{Q}_{L} \lambda_{d} d_{R} h^{*}+\bar{L}_{L} \lambda_{e} e_{R} h^{*}+\cdots$

where $h$ is the only light Higgs doublet and the multiplets $Q, u, d, e$, each with a flavour index and canonically nor- malised kinetic terms, have definite transformation properties under $U(3)_{T} \times U(3)_{\bar{F}}$

$$
\begin{aligned}
& \left(Q_{L}, u_{R}^{*}, e_{R}^{*}\right) \rightarrow V_{T}\left(Q_{L}, u_{R}^{*} \cdot e_{R}^{*}\right), \\
& \left(L_{L}, d_{R}^{*}\right) \rightarrow V_{F}\left(L_{L}, d_{R}^{*}\right),
\end{aligned}
$$

as do the low energy Yukawa matrices $\lambda_{u, d, e}$

$\lambda_{u} \rightarrow V_{T} \lambda_{u}\left(V_{T}\right)^{T}, \quad\left(\lambda_{d}^{T}, \lambda_{e}\right) \rightarrow V_{F}\left(\lambda_{d}^{T}, \lambda_{e}\right)\left(V_{T}\right)^{T}$.

The matrices $\lambda_{u, d, e}$ control at the same time the flavour symmetry properties of every other higher dimensional operator left understood in (13). This is because they are in the same number as the original "spurions" $Y_{u}, Y_{1}, Y_{2}$, with the same transformation properties up to a complex conjugation. For example it is

$\lambda_{u}=\Sigma_{n>0} a_{n} Y_{T}^{*}\left(Y_{T} Y_{T}^{*}\right)^{n}$

where the $a_{n}$ are complex coefficients of order unity or smaller and we neglect terms involving powers of $Y_{1} Y_{1}^{+}, Y_{2} Y_{2}^{+}, Y_{1} Y_{2}^{+}$.

In analogy with the $U(3)^{3}$ case, by $U(3)^{2}$ transformations one can set

$\lambda_{u}=\lambda_{u}^{D}, \quad \lambda_{d}=V \lambda_{d}^{D}$,

where $V$ is again the CKM matrix. On the contrary $\lambda_{e}$ has the form

$\lambda_{e}=V_{e F} \lambda_{e}^{D}\left(V_{e T}\right)^{T}$

where $V_{e F}, V_{e T}$ are fixed unknown unitary matrices.

\section{1 $U(3)^{2}$ and lepton flavour violation}

The presence of two spurions $\lambda_{d}^{T}$ and $\lambda_{e}$ with the same transformations properties under $U(3)^{2}$, one of which dependent on unknown mixing matrices, is the source of potentially large deviations from MFV, particularly from chiralitybreaking down-quark operators. If compared with the current bounds, an even stronger direct constraint arises from lepton flavour violation (LFV) and, more specifically, from the $\mu \rightarrow e+\gamma$ transition.

The relevant operator is

$\frac{c}{\Lambda^{2}} e F_{\mu \nu}\left(\bar{e}_{L} \lambda_{d}^{T} \sigma_{\mu \nu} e_{R}\right) v+$ h.c.

which, in terms of the physical charged leptons (still denoted in the same way), becomes

$\frac{c}{\Lambda^{2}} e F_{\mu \nu}\left(\bar{e}_{L} V_{e F}^{+} \lambda_{d}^{D} V^{T} V_{e T}^{*} \sigma_{\mu \nu} e_{R}\right) v+$ h.c. 
For $\mu \rightarrow e+\gamma$ the likely dominant term is the one proportional to the mass of the $b$-quark

$$
\begin{aligned}
& \frac{c}{\Lambda^{2}} e m_{b} F_{\mu \nu}\left[A_{32}^{*} B_{13}\left(\bar{\mu}_{L} \sigma_{\mu \nu} e_{R}\right)\right. \\
& \left.\quad+A_{31}^{*} B_{23}\left(\bar{e}_{L} \sigma_{\mu \nu} \mu_{R}\right)\right]+ \text { h.c.; } \\
& A=V_{e F}, \quad B=V_{e T}^{+} V,
\end{aligned}
$$

which leads to a transition rate

$$
\Gamma_{\mu \rightarrow e \gamma}=\alpha \frac{m_{\mu}^{3} m_{b}^{2}}{\Lambda^{4}}\left(\left|c A_{32}^{*} B_{13}\right|^{2}+\left|c A_{31}^{*} B_{23}\right|^{2}\right) .
$$

From the current bound on the branching ratio of 5.7 . $10^{-13}[13]$ and using $m_{b}(3 \mathrm{TeV})=2.4 \mathrm{GeV}$, one gets

$$
|c|\left(\left|A_{32}^{*} B_{13}\right|^{2}+\left|A_{31}^{*} B_{23}\right|^{2}\right)^{1 / 2} \lesssim 5 \cdot 10^{-7}\left(\frac{\Lambda}{3 \mathrm{TeV}}\right)^{2} .
$$

Even taking into account a possible loop suppression factor, this is a strong constraint, far beyond the typical MFV bounds. As mentioned a somewhat weaker but still significant constraint on the misalignment of the $\lambda_{d}$ and $\lambda_{e}$ matrices arises from the consideration of chirality-breaking $\Delta B$ or $\Delta S$ transitions. Although still highly significant, the constraint in (23) can be made weaker by a factor $m_{b} / m_{s} \approx 50$ if one assumes that all the elements of $Y_{2}$ in Eq. (10) are at most of order $m_{s}$, which is sufficient to cure the mass relation problem of the single $T Y_{1} \bar{F} H_{\overline{5}}$ coupling.

\section{$4 U(2)^{2}$ and $S U(5)$-unification}

The starting point is again the $S U(5)$-invariant Yukawa Lagrangian

$$
\begin{aligned}
\mathscr{L}_{Y}^{U(2)}= & y_{t} T_{3} T_{3} H_{5}+y_{t} x_{t} \mathbf{T V} T_{3} H_{5}+\mathbf{T} \Delta_{u} \mathbf{T} H_{5} \\
& +y_{b} T_{3} \bar{F}_{3} H_{\overline{5}}+y_{b} x_{b} \mathbf{T V} \bar{F}_{3} H_{\overline{5}} \\
& +\mathbf{T} \Delta_{1} \overline{\mathbf{F}} H_{\overline{5}}+\mathbf{T} \Delta_{2} \overline{\mathbf{F}} H_{45},
\end{aligned}
$$

invariant under

$$
U(2)_{T} \times U(2)_{\bar{F}} \times U(1)_{\bar{F} 3} \equiv U(2)^{2} .
$$

With respect to $U(2)^{2}$ it is

$$
T_{3}=(1,1)_{0}, \quad \bar{F}_{3}=(1,1)_{1}, \quad \mathbf{T}=(2,1)_{0}, \quad \overline{\mathbf{F}}=(1,2)_{0}
$$

with the spurions $\lambda_{b}, \mathbf{V}, \Delta_{u}, \Delta_{1}, \Delta_{2}$ transforming accordingly to keep $\mathscr{L}_{Y}$ invariant.
In analogy with the $U(3)^{2}$ discussion, the low energy Lagrangian in this case assumes the form ${ }^{3}$

$$
\begin{aligned}
& \mathscr{L}_{\text {low energy }}^{U(2)}=\left(y_{t} \bar{Q}_{L 3} u_{R 3}+y_{t} x_{t} \overline{\mathbf{Q}}_{L} \mathbf{V} u_{R 3}+y_{t} x_{t} \bar{Q}_{L 3} \mathbf{V} \mathbf{u}_{R}\right. \\
& \left.+\overline{\mathbf{Q}}_{L} \lambda_{u} \mathbf{u}_{R}\right) h+\left(y_{b} \bar{Q}_{L 3} d_{R 3}+y_{b} x_{b} \overline{\mathbf{Q}}_{L} \mathbf{V} d_{R 3}+\overline{\mathbf{Q}}_{L} \lambda_{d} \mathbf{d}\right) h^{*} \\
& +\left(y_{\tau} \bar{L}_{L 3} e_{R 3}+y_{\tau} x_{\tau} \overline{\mathbf{L}}_{L} \mathbf{V} e_{R 3}+\overline{\mathbf{L}}_{L} \lambda_{e} \mathbf{e}\right) h^{*}+\cdots
\end{aligned}
$$

with self-evident transformation properties under $U(2)^{2}$ of the various fields/spurions. By these same transformations one can set $\mathbf{V}, \lambda_{u}$ and $\lambda_{d}$ as [see Eq. (6)]

$\mathbf{V}=\left(\begin{array}{l}0 \\ \epsilon\end{array}\right), \quad \lambda_{u}=L_{12}^{u} \lambda_{u}^{D}\left(L_{12}^{u}\right)^{T}, \quad \lambda_{d}=\Phi_{L} L_{12}^{d} \lambda_{d}^{D}$,

and $\lambda_{e}$ to the form

$\lambda_{e}=U_{e F} \lambda_{e}^{D}\left(U_{e T}\right)^{T}$

where $U_{e F}, U_{e T}$ are fixed unknown $2 \times 2$ unitary matrices. In $3 \times 3$ flavour space the low energy Yukawa matrices are given by

$$
\begin{aligned}
& Y_{u}=\left(\begin{array}{c:c}
\lambda_{u} & y_{t} x_{t} \mathbf{V} \\
\hdashline y_{t} x_{t} \mathbf{V}^{T} & y_{t}
\end{array}\right), \quad Y_{d}=\left(\begin{array}{c:c}
\lambda_{d} & y_{b} x_{b} \mathbf{V} \\
\hdashline 0 & y_{b}-
\end{array}\right), \\
& Y_{e}=\left(\begin{array}{c:c}
\lambda_{e} & 0 \\
\hdashline y_{\tau} x_{\tau} \overline{\mathbf{V}}^{T} & y_{\tau}
\end{array}\right) .
\end{aligned}
$$

Altogether this means that to a sufficient approximation the low energy Yukawa matrices are diagonalised by the unitary $3 \times 3$ matrices

$Y_{u}=U_{u} Y_{u}^{D} U_{u}^{T}, \quad Y_{d}=U_{d} Y_{d}^{D}, \quad Y_{e}=U_{e L} Y_{e}^{D} U_{e R}$

where

$U_{e L}=\left(\begin{array}{c:c}U_{e F} & 0 \\ \hdashline 0 & 1\end{array}\right) \quad U_{e R}=\left(\begin{array}{c:c}\left(U_{e T}\right)^{T} & 0 \\ \hdashline 0 & 1\end{array}\right) U_{23}(\epsilon)$

with $U_{23}(\epsilon)$ a unitary transformation of order $\epsilon$ in the 2-3 sector. $U_{u}, U_{d}$ are the same diagonalisation matrices as in normal $U(2)^{3}$, with their parameters determined as in $(8,9)$, except that in $U(2)^{3}$ case the matrix $U_{u}$ acts only on the left side of $Y_{u}^{D}$.

Before proceeding, let us note that $U(2)^{2}$, unlike $U(3)^{2}$, makes natural room for the successful relations $m_{b} \approx m_{\tau}$ and $m_{\mu} \approx 3 m_{s}$, valid at unification. This only requires that $\Delta_{1}$ be sufficiently smaller than $\Delta_{2}$ not to undo the last relation

${ }^{3}$ Note a small abuse of notation: here and below the matrices $\lambda_{u, d, e}$ act in the 1-2 flavour space, unlike the case of Sect. 3 where they act on the full 1-2-3 space. 
arising from the coupling to $H_{45}$. At the same time $\Delta_{1}$ must be capable to give the proper relation between $m_{e}$ and $m_{d}$. We assume in the following that all the elements of $\Delta_{1}$ are at most of the order needed to this purpose. This in turn implies that the relative alignment between the $\lambda_{d}$ and $\lambda_{e}^{T}$ matrices is, without any further assumption or tuning, of order $m_{d} / m_{s}$ both on the left and on the right side.

\section{1 $U(2)^{2}$ and LFV}

In analogy with the discussion in the $U(3)^{2}$ case, the presence of two spurions with the same transformation properties in the down and charged lepton sectors is a source of potentially large flavour violations. In the $U(2)^{2}$ case, however, there are two significant differences. As just said, in 1-2 flavour space $\lambda_{d}$ and $\lambda_{e}^{T}$ are misaligned only by relative rotations of the order of $m_{d} / m_{s}$. Furthermore, due to the small $U(2)^{2}$ breaking, the diagonalisation of both $Y_{d}$ and $Y_{e}$ in 2-3 flavour space is obtained by small rotations of the same order $\epsilon$.

Here again the leading constraint comes for the $\mu \rightarrow e \gamma$ transition. The relevant operator is

$\frac{c^{\mu \rightarrow e \gamma}}{\Lambda^{2}} e F_{\mu \nu}\left(\overline{\mathbf{e}}_{L} \lambda_{d}^{T} \sigma_{\mu \nu} \mathbf{e}_{R}\right) v+$ h.c.

only acting in 1-2 flavour space. Therefore, after going to the physical basis, one obtains a transition rate

$\Gamma_{\mu \rightarrow e \gamma}=\alpha \frac{m_{\mu}^{3} m_{s}^{2}}{\Lambda^{4}}\left|c^{\mu \rightarrow e \gamma}\right|^{2}\left(\left|A_{22}^{*} B_{12}\right|^{2}+\left|A_{21}^{*} B_{22}\right|^{2}\right)$,

where this time $A, B$ are the misalignment matrices between $\lambda_{e}$ and $\lambda_{D}^{T}$ in the 1-2 sector, of order $m_{d} / m_{s}$. One gets therefore the bound

$$
\begin{aligned}
& \left|c^{\mu \rightarrow e \gamma}\right|\left(\left|\frac{A_{22}^{*} B_{12}}{m_{d} / m_{s}}\right|^{2}+\left|\frac{A_{21}^{*} B_{22}}{m_{d} / m_{s}}\right|^{2}\right)^{1 / 2} \\
& \lesssim 5 \cdot 10^{-4}\left(\frac{\Lambda}{3 \mathrm{TeV}}\right)^{2},
\end{aligned}
$$

to be compared with the bound in Eq. (23). This is still a significant limit, but now a loop suppression factor of about $10^{-3}$, as illustrated below in a specific example, could be consistent with new particles of $\mathrm{TeV}$ mass.

\subsection{Electric dipole moments}

In $U(2)^{3}$ (as in $U(3)^{3}$ ) one expects EDMs for the quarks, most significantly the ones of the first generation that con- tribute to the neutron EDM. If one includes also the electron EDM, the relevant operators are ${ }^{4}$

$$
\begin{aligned}
& \frac{c_{e}^{E D M} m_{e}}{\Lambda^{2}} e F_{\mu \nu}\left(\bar{e}_{L} \sigma_{\mu \nu} e_{R}\right), \quad \frac{c_{u}^{E D M} m_{u}}{\Lambda^{2}} e F_{\mu \nu}\left(\bar{u}_{L} \sigma_{\mu \nu} u_{R}\right), \\
& \frac{c_{d}^{E D M} m_{d}}{\Lambda^{2}} e F_{\mu \nu}\left(\bar{d}_{L} \sigma_{\mu \nu} d_{R}\right)
\end{aligned}
$$

with $c_{e, u, d}^{E D M}$ generally complex in the physical basis. From the current bounds on the electron and neutron EDMs, respectively $d_{e}<8.7 \times 10^{-29} e \cdot \mathrm{cm}[14]$ and $d_{n}<2.9 \times 10^{-26} e \cdot \mathrm{cm}$ [15], one gets the corresponding limits on the imaginary parts of these coefficients [16-18]

$$
\begin{aligned}
& \left|\operatorname{Im}\left(c_{d}^{E D M}\right)\right| \lesssim 5.6 \times 10^{-3}\left(\frac{\Lambda}{3 \mathrm{TeV}}\right)^{2}, \\
& \left|\operatorname{Im}\left(c_{u}^{E D M}\right)\right| \lesssim 1.6 \times 10^{-2}\left(\frac{\Lambda}{3 \mathrm{TeV}}\right)^{2}, \\
& \left|\operatorname{Im}\left(c_{e}^{E D M}\right)\right| \lesssim 8 \times 10^{-5}\left(\frac{\Lambda}{3 \mathrm{TeV}}\right)^{2} .
\end{aligned}
$$

In the $U(2)^{2}$ case, one expects additional contributions to the coefficients of the operators in line (36)

$$
\begin{aligned}
c_{e}^{E D M} m_{e} & \rightarrow c_{e}^{E D M} m_{e}+\tilde{c}_{e}^{E D M} m_{d}, \\
c_{u}^{E D M} m_{u} & \rightarrow c_{u}^{E D M} m_{u}+\tilde{c}_{u}^{E D M} m_{t}\left|V_{b u}\right|^{2},
\end{aligned}
$$

whereas the dipole of the down quark receives negligible corrections. For the electron the additional contribution comes from the same type of operator as in Eq. (33), whereas for the up quark it is due to the fact that in $U(2)^{2}$ both $\mathbf{Q}_{L}$ and $\mathbf{u}_{R}$ transform under the same $U(2)$ group factor or, differently stated, that the diagonalisation matrix $U_{u}$ is present on both sides of $Y_{u}^{D}$ in Eq. (31). Barring cancellations this gives the limits

$$
\begin{gathered}
\left|\operatorname{Im}\left(\tilde{c}_{u}^{E D M}\right)\right| \lesssim 1.2 \times 10^{-2}\left(\frac{\Lambda}{3 \mathrm{TeV}}\right)^{2} \\
\left|\operatorname{Im}\left(\tilde{c}_{e}^{E D M}\right)\right| \lesssim 1.6 \times 10^{-5}\left(\frac{\Lambda}{3 \mathrm{TeV}}\right)^{2} .
\end{gathered}
$$

\section{$4.3 U(2)^{2}$ and quark flavour violation}

The counterpart in the down-quark sector of the chiralitybreaking effect in $\mu \rightarrow e \gamma$ of Sect. 4.1 is due to the operator

\footnotetext{
${ }^{4}$ For brevity we do not discuss the chromo-magnetic dipole operators for the up and down quarks, but they lead to similar bounds on the corresponding coefficients. The contribution of the charm chromo-electric dipole, $c_{c}^{C E D M} m_{c} / \Lambda^{2}$, to the three gluon Weinberg CP-violating operator gives also a significant bound $\left|\operatorname{Im}\left(c_{c}^{C E D M}\right)\right| \lesssim 3 \times 10^{-2}(\Lambda / 3 \mathrm{TeV})^{2}$ [19]. Note also that along this line by $e, u, d$ we mean specifically the first generation particles and not the flavour triplets as in Sect. 3.
} 
Table 1 Upper bounds on the coefficients of the operators discussed in the text, normalised to $\Lambda=3 \mathrm{TeV}$

\begin{tabular}{lllllll}
\hline Observable & $\mu \rightarrow e \gamma$ & $\mathrm{e} \mathrm{EDM}$ & $\mathrm{u}$ EDM & $\mathrm{d}$ EDM & $\epsilon^{\prime}$ & $A_{C P}^{\Delta C=1}$ \\
Coefficient & $\left|c^{\mu \rightarrow e \gamma}\right|$ & $\left|\operatorname{Im}\left(\tilde{c}_{e}^{E D M}\right)\right|$ & $\left|\operatorname{Im}\left(\tilde{c}_{u}^{E D M}\right)\right|$ & $\left|\operatorname{Im}\left(c_{d}^{E D M}\right)\right|$ & $\left|c^{\Delta S=1} \sin \phi\right|$ & $\left|c^{\Delta C=1}\right|$ \\
Upper bound & $5 \times 10^{-4}$ & $1.6 \times 10^{-5}$ & $1.2 \times 10^{-2}$ & $5.6 \times 10^{-3}$ & $6.5 \times 10^{-2}$ & 0.2 \\
\hline
\end{tabular}

$\frac{c^{\Delta S=1}}{\Lambda^{2}} g_{s} G_{\mu \nu}^{a}\left(\overline{\mathbf{d}}_{L} \lambda_{e}^{T} \sigma_{\mu \nu} T^{a} \mathbf{d}_{R}\right) v+$ h.c.

By a similar line of reasoning to the one that leads to the limit in Eq. (35) and following the analyses of [20,21], the effect of this operator on the parameter $\epsilon^{\prime}$ sets the bound

$\left|c^{\Delta S=1}\right||\sin \phi| \lesssim 6 \times 10^{-2}\left(\frac{\Lambda}{3 \mathrm{TeV}}\right)^{2}$

where the factor $\sin \phi$ is there to recall the role of phases, in general a combination of them, in this effect and we have set $\left|A_{12}\right| \approx\left|B_{12}\right| \approx m_{d} / m_{s}$.

In the up-quark sector the Yukawa couplings in $3 \times 3$ flavour space have the form $Y_{u}=U_{u} Y_{u}^{D} U_{u}^{T}$ with $U_{u}=$ $U_{12} U_{23}$. Similarly one can write down a $U(2)^{2}$-invariant dipole operator with the flavour structure $D_{u}=\tilde{U}_{u} D_{u}^{D} \tilde{U}_{u}^{T}$ and $\tilde{U}_{u}=U_{12} \tilde{U}_{23}$. The point is that the unitary transformations in the 1-2 sector are the same in the two cases whereas $U_{23}$ and $\tilde{U}_{23}$, although both of order $\epsilon$, are different from each other. In the physical basis, therefore, $D_{u}$ keeps to a good approximation the same form, except for a different $U_{23}$ transformation, still of order $\epsilon$. This is the source both of the correction to the up-quark EDM, discussed in Sect. 4.2, and of the chirality-breaking $\Delta C=1$ operator proportional to $m_{t}$

$$
\begin{aligned}
& \frac{c^{\Delta C=1} m_{t}}{\Lambda^{2}} V_{u b} V_{c b}^{*} g_{s} G_{\mu \nu}^{a}\left[\left(\bar{u}_{L} \sigma_{\mu \nu} T^{a} c_{R}\right)\right. \\
& +\left(\bar{u}_{R} \sigma_{\mu \nu} T^{a} c_{L}\right]+\text { h.c. }
\end{aligned}
$$

Following [21,22], the current limit on direct CP violation in $D \rightarrow \pi \pi, K K$ decays gives the bound

$$
\left|c^{\Delta C=1}\right| \frac{\sin \left(\delta-\phi^{\Delta C=1}\right)}{\sin \delta} \lesssim 0.2\left(\frac{\Lambda}{3 \mathrm{TeV}}\right)^{2}
$$

Before closing this section we summarise in Table 1 the bounds on the coefficients of the different operators normalised to a scale $\Lambda=3 \mathrm{TeV}$. The bounds on $\operatorname{Im}\left(c_{e}^{E D M}\right)$ and $\operatorname{Im}\left(c_{u}^{E D M}\right)$ are $8 \times 10^{-5}$ and $1.2 \times 10^{-2}$ respectively. The bounds on the coefficients of the other FCNC operators are at the typical $10^{-1} \div 10^{-2}$ level $[6,12]$, depending on their phases, and are the same in $U(2)^{2}$ or $U(3)^{2}$.

\section{$5 U(2)^{2}$ in supersymmetry}

The picture that emerges from the previous sections is that $U(2)^{2}$ gives rise to several new effects than the ones normally considered in MFV, with the relevant feature, as in MFV, that their flavour structure, both in the quark and, more interestingly, in the charged lepton sector, is always controlled by the CKM matrix elements. It is this feature that allows to make quantitative considerations. $^{5}$

There are two good reasons that make it relevant to see the impact of the above considerations in the special case of supersymmetry. First of all is the obvious connection of supersymmetry with gauge coupling unification. No less important, however, is the consideration of the bounds in Table 1. Unless the various coefficients include a loop suppression factor, as in the case of supersymmetry, one can interpret them as quite strong lower bounds on the scale $\Lambda$, much above the few $\mathrm{TeV}$ range. In turn this appears in contrast with our original motivation of understanding the compliance with the flavour constraints of new particles with masses in the $\mathrm{TeV}$ range.

The model we consider is a generic supersymmetric $S U(5)$-theory with a Yukawa superpotential that gives rise to $\mathscr{L}_{Y}^{U(2)}$ as in Eq. (24) and with soft supersymmetry-breaking terms generated by supergravity. In the flavour sector the entire theory is invariant under $U(2)^{2}$ as in Eqs. (25) and (26). On this basis we shall consider the low energy theory in two different ways. We implement the general case as discussed above or we take universal $A$-terms at least when restricted to the 1-2 sector.

At low energy flavour-changing effects are present in the Yukawa couplings, in the $A$-terms and in the squared masses for squarks and leptons. The Yukawa couplings $Y_{u, d, e}$, with the usual meaning of the angle $\beta$,

$$
\mathscr{L}_{Y}=v \sin \beta \bar{u}_{L} Y_{u} u_{R}+v \cos \beta\left(\bar{d}_{L} Y_{d} d_{R}+\bar{e}_{L} Y_{e} e_{R}\right)+\text { h.c. }
$$

take the form of Eq. (30). The $A$-terms

$$
\mathscr{L}_{A}=v \sin \beta \tilde{u}_{L}^{+} A_{u} \tilde{u}_{R}+v \cos \beta\left(\tilde{d}_{L}^{+} A_{d} \tilde{d}_{R}+\tilde{e}_{L}^{+} A_{e} \tilde{e}_{R}\right)+h . c .
$$

\footnotetext{
$\overline{5}$ This same feature is achieved in [23], where, however, it rests on a superpotential as in (10) without the coupling to $H_{45}$, necessary for a realistic description of fermion masses.
} 


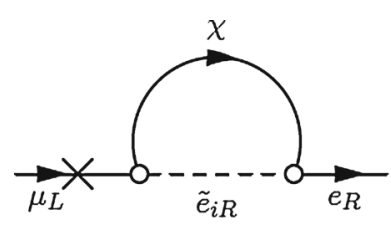

(a)

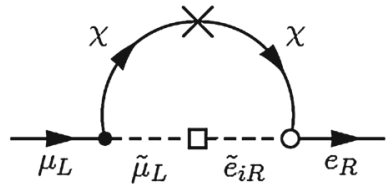

(b)

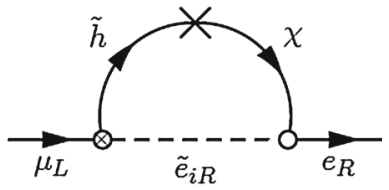

(c)

Fig. 1 Diagrams contributing to the $\mu \rightarrow e \gamma$ amplitude. Crosses denote a chirality flip. White circles denote flavour-changing interaction vertices controlled by $U_{e}$. The white square in Fig. $1 \mathrm{~b}$ is the $A_{e}$-insertion

have an analogous structure

$$
\begin{aligned}
& A_{u}=\left(\begin{array}{c:c}
a_{u} \lambda_{u} & y_{t} a_{u t} \mathbf{V} \\
\hdashline y_{t} a_{u t} \overline{\mathbf{V}}^{T} & a_{t} y_{t}
\end{array}\right), \\
& A_{d}=\left(\begin{array}{c:c}
a_{d 1} \lambda_{d}+a_{d 2} \lambda_{e}^{T} & y_{b} a_{d b} \mathbf{V} \\
\hdashline 0 & a_{b} y_{b}
\end{array}\right),
\end{aligned}
$$

and

$$
A_{e}=\left(\begin{array}{c:c}
a_{e 1} \lambda_{e}+a_{e 2} \lambda_{d}^{T}: & 0 \\
\hdashline y_{\tau} \bar{a}_{e \tau}-\mathbf{V}^{T} \bar{d}^{-} & a_{\tau} \bar{y}_{\tau}^{-}
\end{array}\right)
$$

where the various $a$-factors are mass terms of similar order of magnitude, related to the low energy scale of effective supersymmetry breaking. Finally the squared masses have two different forms. Up to negligibly small terms quadratic in $\lambda_{e, d, u}$, the squared masses for $\tilde{L}$ and $\tilde{d}_{R}$ are diagonal and degenerate in 1-2 sector, whereas the mass terms for $\tilde{e}_{R}, \tilde{u}_{R}$ and $\tilde{Q}$ have contributions controlled by the spurion $\mathbf{V}$, i.e.

$M_{\tilde{e}}^{2}=\left(\begin{array}{c:c}m_{e 1}^{2} \mathbf{1} & m_{e 12}^{2} \mathbf{V} \\ \hdashline m_{e 12}^{2}{ }_{\overline{\mathbf{V}}} T_{1} & m_{e 3}^{2}-\end{array}\right)$,

and similar for $M_{\tilde{Q}}^{2}, M_{\tilde{u}}^{2}$.

\section{$5.1 \mu \rightarrow e+\gamma$}

To discuss $\mu \rightarrow e \gamma$ it is convenient to go to the basis where $Y_{e}$ and $M_{\tilde{e}}^{2}, M_{\tilde{L}}^{2}$ are diagonal, i.e. the physical basis for the charged leptons but not for the sleptons, since $A_{e}$ is still nondiagonal. In this basis both the bino, $\chi$, and the neutral higgsino have flavour-changing interactions with the $\left(e_{R}, \tilde{e}_{R}\right)$ multiplets

$$
\mathscr{L}_{\chi, \tilde{h}}=\sqrt{2} g^{\prime} \chi\left(\tilde{e}_{R}^{+} U_{e}^{+} e_{R}\right)+v \cos \beta \tilde{h}\left(\tilde{e}_{R}^{+} U_{e}^{+} Y_{e}^{D} e_{L}\right)+h . c .
$$

where, as in the previous examples, $U_{e}$ is as $U_{e R}$ in Eq. (32), except for a different unitary transformation in the 23 sector, although still of order $\epsilon$. Furthermore in the 1-2 sector $U_{e T}=U_{d}$ up to a small misalignment of order $m_{d} / m_{s}$. Hence the flavour violation in these interactions is controlled by the CKM angles. Note also the non-degeneracy of relative order $\epsilon^{2}$ between the first two generations of right-handed leptons, which will play a role in the following.

Let us now look at the $A_{e}$-term in Eq. (45). If the $A$-terms were universal, at least in the 1-2 sector, one would have no $\lambda_{d}^{T}$ term in (46) and (47) and, in the basis under consideration, we would have

$A_{e} \rightarrow\left(\begin{array}{c:c}a_{e 1} \lambda_{e}^{D} & 0 \\ \hdashline 0 & a_{\tau} y_{\tau}\end{array}\right) U_{e}$

On the contrary, in the general case, in which both $\lambda_{e}$ and $\lambda_{d}^{T}$ are present, it is the latter that dominates. Therefore, in this case we have

$A_{e} \rightarrow \hat{U}_{12}\left(m_{d} / m_{s}\right)\left(\begin{array}{c:c}a_{e 2} \lambda{ }_{d}^{D} & 0 \\ \hdashline 0 & a_{\tau} y_{\tau}\end{array}\right) U_{12}\left(m_{d} / m_{s}\right) U_{e}$

where on both sides of the diagonal term there appear two unitary 1-2 transformations of order $m_{d} / m_{s}$, representing precisely the misalignment of $\lambda_{d}^{T}$ with $\lambda_{e}$.

The diagrams that contribute to $\mu \rightarrow e \gamma$ are shown in Fig. 1. Based on Eqs. (49) and (50) (with $A$-terms universal) the only flavour-changing matrix present in these interactions is $U_{e}$. As such, a GIM-like cancellation takes place, controlled by the non-degeneracy of the charged sleptons, of relative order unity between the third and the first two generations and of relative order $\epsilon^{2}$ within the first two generations. As a consequence the $\mu \rightarrow e \gamma$ amplitude receives contributions proportional to $m_{\mu} U_{e}^{*}(\mu \tau) U_{e}(e \tau)$ (a 1-2/3 effect) or to $m_{\mu} \epsilon^{2} U_{e}^{*}(\mu \mu) U_{e}(e \mu)$ (a $1-2$ effect), both equal to $m_{\mu} V_{t s}^{*} V_{t d}$ up to a factor of order unity. In the case of a general $A_{e}$-term the presence of the misalignment matrices in Eq. (51) inhibits the GIM-like cancellation in the diagram of Fig. 1b, which then becomes the dominant contribution to the amplitude, proportional to $m_{d}$ and mediated by exchanges of sleptons of the first two generations.

Representative values for the size of these effects, taken incoherently, and normalised to the current limit, $B R(\mu \rightarrow$ $e \gamma)<5.3 \times 10^{-13}$ [13], are shown in Figs. 2 and 3 both for general and universal $A_{e}$-term. Consistently with this bound, the largest possible value of $B R(\tau \rightarrow \mu \gamma)$ can be reached with a universal $A_{e}$-term, at $10^{-9}$ level. One should remember the order one unknown factor in front of each of these amplitudes. 


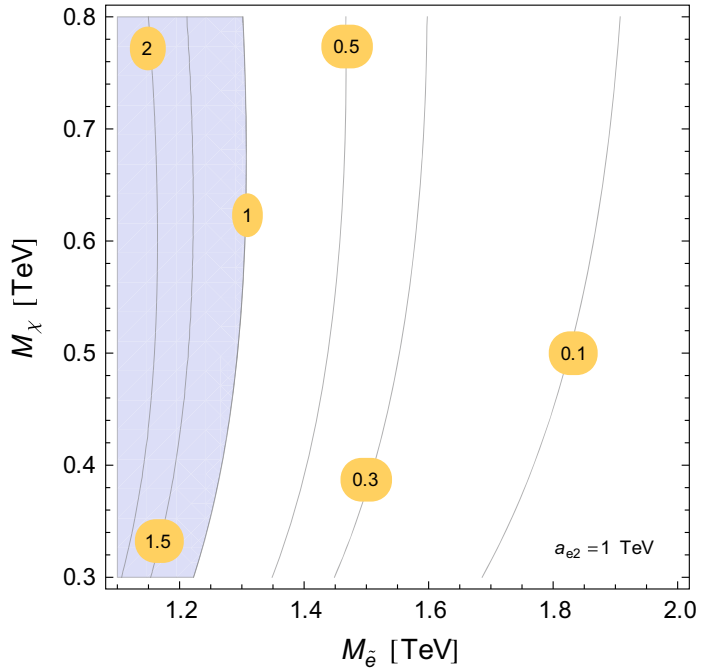

Fig. $2 B R(\mu \rightarrow e \gamma)$ normalised to the current bound, $B R<5.3 \times$ $10^{-13}$, with non-universal $A$-terms for a right-handed selectron mass of the first two generations $M_{\tilde{e}}$ and neutralino mass $M_{\chi}=\mu$

\subsection{Electron EDM}

As seen in Table 1, the electron EDM is potentially capable of providing the strongest limit, although generally dependent on more than one unknown phase. In supersymmetry a wellknown effect that arises from interactions not included in Eqs. (44) or (45), since it is not related to flavour-changing phenomena, is due to a chargino-sneutrino one loop diagram. From the current experimental limit [15] and $\left|M_{\chi}\right|=|\mu|$ on obtains the bound on the mass of the sneutrino of the first generation [24]

$m_{\tilde{v}}>17 \mathrm{TeV} \cdot\left(\sin \phi_{\mu} \tan \beta\right)^{1 / 2}$.

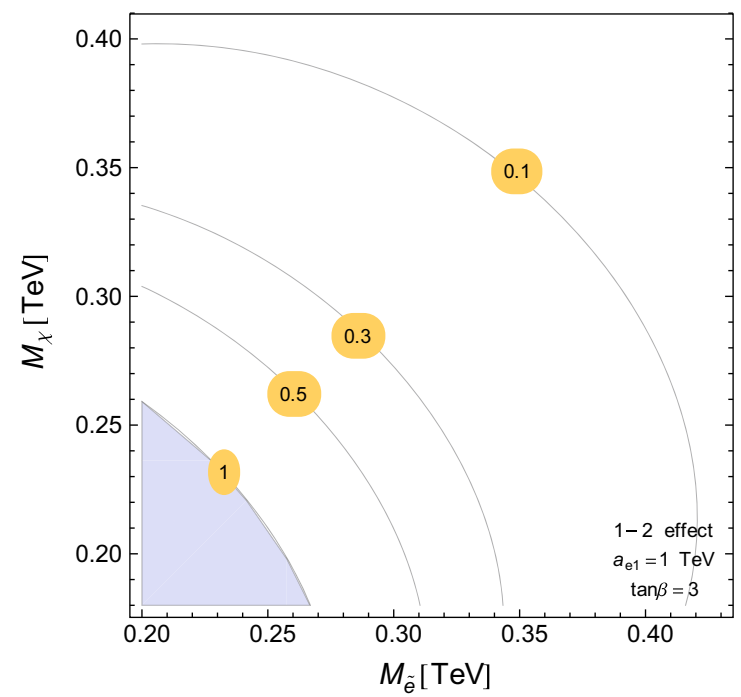



Fig. 4 Electron EDM normalised to the current bound, $d_{e}<8.7 \times$ $10^{-29} e \cdot \mathrm{cm}$, with non-universal $A$-terms and $M_{\tilde{e}}, M_{\chi}$ as in Fig. 2

The interactions in Eqs. (44) or (45) also contribute to the electron EDM by diagrams analogous to the ones in Fig. 1, again with a distinction between universal or non universal $A_{e}$-term. Representative values for the size of these effects are shown in Figs. 4 and 5 for maximal values of the relevant phases and some choice of the other parameters, as indicated.

\section{Summary and conclusions}

The effort to increase the precision of current flavour tests of the SM, now at the $10 \div 30 \%$ level, is a strongly motivated task of particle physics in itself. At the same time this effort

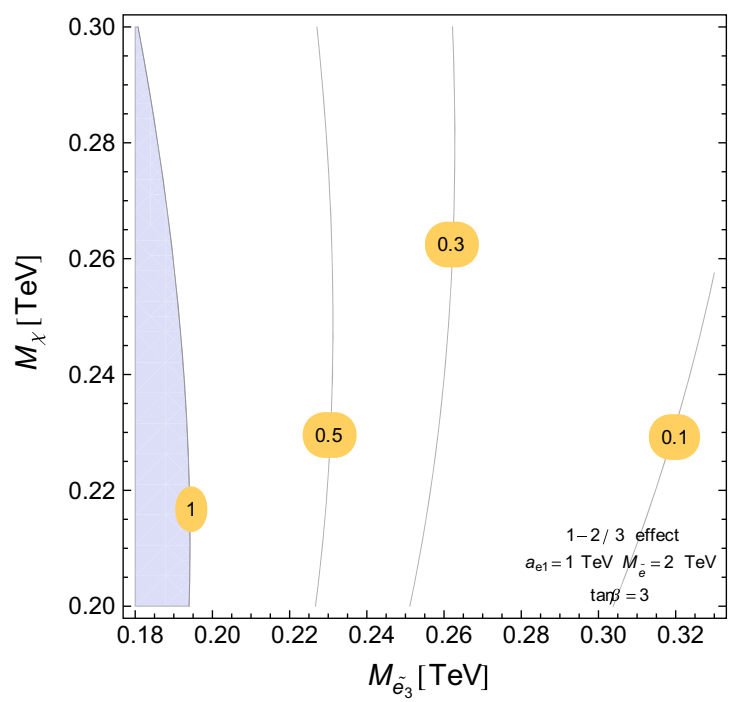

Fig. 3 As in Fig. 2 with universal $A$-terms for a right-handed selectron mass $M_{\tilde{e}}$ of the first two generations (1-2 effect, left) and for a right-handed selectron mass $M_{\tilde{e}_{3}}$ of the first two generations (1-2/3 effect, right) 

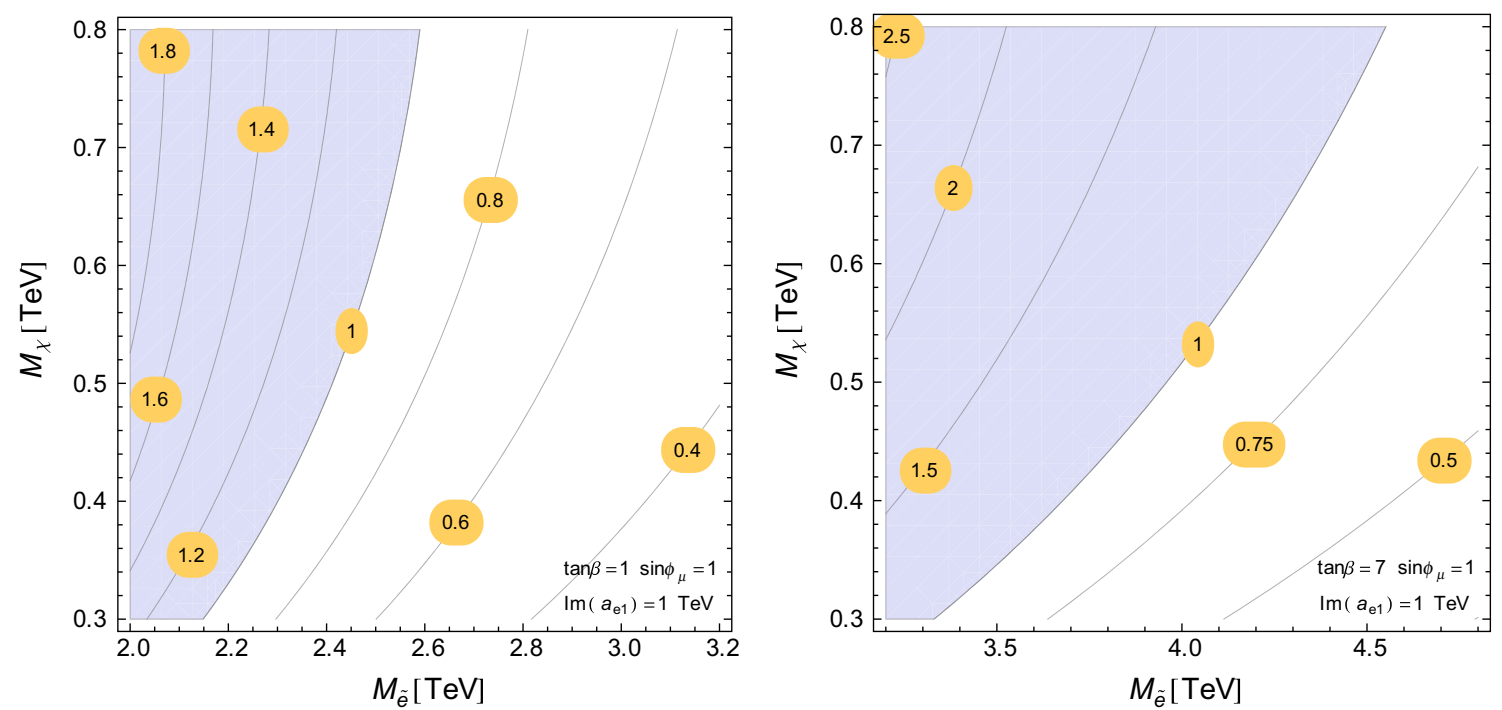

Fig. 5 As in Fig. 4 with universal $A$-terms for $\tan \beta=1$ (left) and $\tan \beta=7$ (right)

could give indirect signals of the existence of new particle at the $\mathrm{TeV}$ scale, complementary to the potentiality of their direct search in high energy collisions. Although not exclusively, nevertheless a strong basis for this statement is the possibility that MFV be at work in some extension of the SM. Especially in its weak form, based on the $U(2)^{3}$ flavour group, phenomenological MFV can explain the absence of new signals so far, while making plausible their emergence in foreseen flavour physics experiments.

While MFV has a predictive content in the quark sector, this is relatively less the case when one tries to extend it to the lepton sector, due to the uncertainties related to the description of neutrino masses. To overcome this problem here we have proposed a predictive scheme based on extending MFV considerations to $S U$ (5)-unification. As far as the quark sector is concerned, weak MFV can be made consistent with $S U$ (5)-unification without introducing new strong constraints, even though some interesting CP-violating effects appear both through $\Delta S=1$ and $\Delta C=1$ chirality-breaking operators. In the charged lepton sector, on the other hand, one predicts flavour violations with intensities also controlled, to a good approximation, by the CKM mixing angles. From a general EFT point of view Table 1 is an effective summary of our findings. As shown there, not unexpectedly, the current limits on $\mu \rightarrow e \gamma$ as on the electron EDM represent strong constraints.

Although not exclusively, supersymmetry is the obvious arena where these considerations might be of relevance. For this reason we have considered their implementation in a realistic supersymmetric $S U(5)$-theory with soft supersymmetry-breaking terms generated by supergravity. Without any extra assumption $\mu \rightarrow e \gamma$ and the electron $\mathrm{EDM}$ with maximal $\mathrm{CP}$-violating phases require charged sleptons of the first two generations in the $1 \div 3 \mathrm{TeV}$ range, as shown in Figs. 2 and 4. Sleptons of the first two generations in the few hundred $\mathrm{GeV}$ range can be made compatible with the flavour scheme proposed here provided the $A$-terms are universal and the $\mathrm{CP}$-violating phases contributing to the electron EDM are not maximal. Third generation sleptons and neutralinos in the few hundred $\mathrm{GeV}$ range are in any case consistent with present bounds. This is illustrated in Figs. 3 and 5. The weaker bound on the mass of the third generation leptons comes from the fact that in any event the communication between them and the first two lepton generations is controlled by the small CKM matrix elements.

Acknowledgments R.B. thanks Dario Buttazzo and Filippo Sala for discussions at the early stage of this work and Gino Isidori and David Straub for useful exchanges. F.S. thanks Diptimoy Ghosh for useful discussions. This work is supported in part by the European Programme "Unification in the LHC Era", contract PITN-GA-2009-237920 (UNILHC) and by MIUR under the contract 2010YJ2NYW-010.

Open Access This article is distributed under the terms of the Creative Commons Attribution 4.0 International License (http://creativecomm ons.org/licenses/by/4.0/), which permits unrestricted use, distribution, and reproduction in any medium, provided you give appropriate credit to the original author(s) and the source, provide a link to the Creative Commons license, and indicate if changes were made. Funded by SCOAP $^{3}$.

\section{References}

1. B. Grinstein, V. Cirigliano, G. Isidori, M.B. Wise, Nucl. Phys. B 763, 35 (2007). arXiv:hep-ph/0608123

2. R.S. Chivukula, H. Georgi, Phys. Lett. B 188, 99 (1987)

3. L.J. Hall, L. Randall, Phys. Rev. Lett. 65, 2939 (1990)

4. G. D'Ambrosio, G.F. Giudice, G. Isidori, A. Strumia, Nucl. Phys. B 645, 155 (2002). arXiv:hep-ph/0207036 
5. R. Barbieri, G. Isidori, J. Jones-Perez, P. Lodone, D.M. Straub, Eur. Phys. J. C 71, 1725 (2011). arXiv:1105.2296 [hep-ph]

6. R. Barbieri, D. Buttazzo, F. Sala, D.M. Straub, JHEP 1207, 181 (2012). arXiv:1203.4218 [hep-ph]

7. V. Cirigliano, B. Grinstein, G. Isidori, M.B. Wise, Nucl. Phys. B 728, 121 (2005). arXiv:hep-ph/0507001

8. S. Davidson, F. Palorini, Phys. Lett. B 642, 72 (2006). arXiv:hep-ph/0607329

9. G.C. Branco, A.J. Buras, S. Jager, S. Uhlig, A. Weiler, JHEP 0709, 004 (2007). arXiv:hep-ph/0609067

10. M.B. Gavela, T. Hambye, D. Hernandez, P. Hernandez, JHEP 0909 , 038 (2009). arXiv:0906.1461 [hep-ph]

11. T. Feldmann, T. Mannel, Phys. Rev. Lett. 100, 171601 (2008). arXiv:0801.1802 [hep-ph]

12. R. Barbieri, D. Buttazzo, F. Sala, D.M. Straub, JHEP 1405, 105 (2014). arXiv:1402.6677 [hep-ph]

13. J. Adam et al. [MEG Collaboration], Phys. Rev. Lett. 110, 201801 (2013). arXiv:1303.0754 [hep-ex]

14. J. Baron et al. [ACME Collaboration], Science 343, 269 (2014). arXiv:1310.7534 [physics.atom-ph]
15. C.A. Baker, D.D. Doyle, P. Geltenbort, K. Green, M.G.D. van der Grinten, P.G. Harris, P. Iaydjiev, S.N. Ivanov et al., Phys. Rev. Lett. 97, 131801 (2006). arXiv:hep-ex/0602020

16. M. Pospelov, A. Ritz, Ann. Phys. 318, 119 (2005). arXiv:hep-ph/0504231

17. J. Engel, M.J. Ramsey-Musolf, U. van Kolck, Prog. Part. Nucl. Phys. 71, 21 (2013). arXiv:1303.2371 [nucl-th]

18. T. Bhattacharya, V. Cirigliano, R. Gupta, H.W. Lin, B. Yoon, arXiv:1506.04196 [hep-lat]

19. F. Sala, JHEP 1403, 061 (2014). arXiv:1312.2589 [hep-ph]

20. P. Mertens, C. Smith, JHEP 1108, 069 (2011). arXiv:1103.5992 [hep-ph]

21. R. Barbieri, D. Buttazzo, F. Sala, D.M. Straub, JHEP 1210, 040 (2012). arXiv:1206.1327 [hep-ph]

22. G. Isidori, J.F. Kamenik, Z. Ligeti, G. Perez, Phys. Lett. B 711, 46 (2012). arXiv:1111.4987 [hep-ph]

23. R. Barbieri, L.J. Hall, A. Strumia, Nucl. Phys. B 445, 219 (1995). arXiv:hep-ph/9501334

24. R. Barbieri, P. Lodone, D.M. Straub, JHEP 1105, 049 (2011). arXiv:1102.0726 [hep-ph] 